\title{
Actitudes hacia el Cálculo Diferencial e Integral: Caracterización de Estudiantes Mexicanos del Nivel Medio Superior
}

\author{
Attitudes towards Differential and Integral Calculus: Characterization of \\ Mexican High School Students
}

\author{
Gustavo Daza* \\ Beatríz Garza ${ }^{* *}$
}

\begin{abstract}
Resumen
Este artículo presenta los resultados de un estudio con estudiantes de la Escuela de Bachilleres Salvador Allende de la Universidad Autónoma de Querétaro, México, que objetivó identificar y caracterizar las actitudes de los estudiantes hacia el Cálculo diferencial e integral. Se utilizó como referente teórico la Teoría de las Actitudes (MANN, 1981). Se utilizó una metodología mixta y se aplicó, como instrumentos de recolección de datos, un cuestionario tipo Likert -alfa $0.807-$ a 149 estudiantes y una entrevista semiestructurada a veintiocho estudiantes. Los hallazgos colocaron en evidencia, por un lado, el rechazo de un grupo significativo de estudiantes hacia el Cálculo como disciplina curricular; y por otro, la manifestación predominante de sentimientos negativos hacia: la metodología de enseñanza, el examen escrito como forma predominante de evaluación, las actividades de aprendizaje, la actitud del maestro en el aula, entre otros aspectos inherentes al proceso escolar. El análisis de los datos obtenidos puede auxiliar en la comprensión de algunas dificultades de los estudiantes en el proceso de enseñanza y aprendizaje de las Matemáticas, así como de las implicaciones de la actitud negativa hacia esta disciplina para el alcance de los objetivos curriculares.
\end{abstract}

Palabras clave: Teoría de las actitudes. Actitudes hacia el Cálculo. Enseñanza del Cálculo. Aprendizaje del Cálculo. Estudiantes de bachillerato.

\begin{abstract}
This article presents the results of a study with students from the Salvador Allende High School of the Autonomous University of Queretaro, Mexico. The goal of the research was to identify and characterize the attitudes of students towards the differential and integral Calculus. We used as theoretical reference the Theory of Attitudes (Mann, 1981). The methodology applied was mixed and a Likert questionnaire -alpha 0.807- was applied as data collection instrument to 149 students and a semi-structured interview to 28 students. The findings show a high degree of student rejection towards the subject of Calculus, and the predominant manifestation of negative feelings to: the teaching methodology, the written exam as the predominant form of assessment, the learning activities, the teacher's attitude in the classroom, among other aspects inherent to the educational process. The analysis of the data obtained can help in the understanding of learning and teaching difficulties, as well as the implications of the negative attitude towards this discipline for achieving curriculum goals.
\end{abstract}

\footnotetext{
* Doctora en Pedagogía por la Universidad Nacional Autónoma de México (UNAM), DF, México. Docente investigadora de tiempo completo en la Universidad Autónoma de Querétaro (UAQ), en la Facultad de Salud, Querétaro, México. Coordinadora de la Maestría en Enseñanza de Estudios Literarios de la Facultad de Lenguas y Letras en la misma universidad. Dirección postal: Circuito Puerta del Sol número 8 Privada del Risco casa 47 Fraccionamiento Puerta Real, Corregidora Querétaro, México C.P. 76910. E-mail: bgarza62@ gmail.com .

${ }^{* *}$ Maestro en Ciencias de la Educación por la Universidad Autónoma de Querétaro (UAQ), Querétaro, México. Maestro en Procesos Socioeducativos y Prácticas Escolares por la Universidad Federal de São João del Rei (UFSJ), São João del Rei, Brasil. Docente en la Institución Educativa Alfonso López Pumarejo de Valledupar, Cesar, Colombia. Dirección postal: Mz $22 \mathrm{~N}^{\circ} 19$, Altos de Comfacesar, Valledupar, Cesar, Colombia. C. P. 200005. E-mail: dazgustavo@gmail.com.
} 
Keywords: Theory of Attitudes. Students' attitudes towards Calculus course. Teaching of Calculus. Learning of Calculus. High School Students.

\section{Introducción}

El presente artículo está organizado de la siguiente manera. En la segunda sección se sitúa al lector en el contexto que suscita la problematización de las actitudes en los procesos de enseñanza y aprendizaje de las matemáticas. Asimismo, se presenta, de forma breve, algunos antecedentes de esta investigación y se muestra la pertinencia de investigar el constructo actitud con miras a comprender desde una perspectiva subjetiva el desempeño académico del estudiante. Además, se expone el referente teórico en el que se sustenta este estudio.

En la tercera sección se describen los procedimientos metodológicos implementados para el alcance de los objetivos, se indican los participantes, los instrumentos aplicados y el procedimiento llevado a cabo. En la cuarta sección se presentan los principales resultados obtenidos a partir del análisis estadístico descriptivo del cuestionario y del Análisis de Contenido de los discursos enunciados en la entrevista. En la quinta sección se lleva a cabo una discusión de los resultados obtenidos. Finalmente, en la sexta sección se presentan algunas consideraciones finales a partir de la reflexión de los resultados.

El estudio es derivado de una investigación para obtener el grado de maestría, y problematizamos la relación existente entre las actitudes del estudiante hacia las Matemáticas y su desenvolvimiento en esta materia, mediante la caracterización de las actitudes hacia el cálculo diferencial e integral de un grupo de estudiantes de bachillerato del sistema público educativo mexicano.

El Cálculo Diferencial e Integral es una de las materias obligatorias del plan de estudio de educación media superior y su importancia tiene distintas aristas. Entre ellas se destaca que durante conversaciones exploratorias con algunos estudiantes de bachillerato, estos percibieron la materia como muy compleja por su nivel de abstracción, pero a su vez, la consideraron como una de las más importantes del bachillerato debido a que es una de las más difíciles de aprobar.

En ese sentido, Vrancken et al. (2006) afirman que la enseñanza del Cálculo constituye uno de los mayores desafíos de la educación actual, ya que su aprendizaje trae aparejado numerosas dificultades relacionadas con un pensamiento de orden superior en el que se encuentran implicados procesos como la abstracción, el análisis, la demostración etc. 
En esa misma lógica, según Artigue (1998) las investigaciones didácticas desarrolladas han mostrado que los estudiantes presentan dificultades fuertes y persistentes con el campo conceptual del Análisis. Asimismo, la autora enfatiza que esas dificultades tienen orígenes diversos y pueden categorizarse en: a) dificultades ligadas a la complejidad matemática de los objetos básicos de este campo conceptual: los números reales, las funciones y las sucesiones, objetos que están siempre en fase de construcción cuando se empieza la enseñanza del Análisis; b) dificultades ligadas a la conceptualización de la noción de límite, que es la noción central del campo, y a su dominio técnico; c) dificultades ligadas a la necesaria ruptura con modos característicos de pensamiento del funcionamiento algebraico.

Por otro lado, en el campo de la Educación Matemática, diversos estudios han expuesto preocupación por mejorar la práctica docente con la implementación de estrategias pedagógicas dirigidas a apoyar la superación de las dificultades de los estudiantes en la comprensión de conceptos tales como Límite, Derivadas e Integral (GÜICHAL et al., 2005; ÁlVAREZ; COLORADO; OSPINA， 2013; IRAZOQUI; MEDINA， 2013; VEGA; CARRILLO; SOTO, 2014, GONZÁLEZ; RADILLO, 2014).

Asimismo, se encontraron investigaciones con el interés en aportar conocimiento sobre cómo se llevan a cabo los procesos de enseñanza y aprendizaje del Cálculo - enfoques y/o modelos de enseñanza, obstáculos epistemológicos, problemas cognitivos y didácticos proporcionando insumos para la discusión y la reflexión acerca de los procesos implicados en la comprensión de los conceptos, así como de las dificultades emergentes tanto en su aplicación como en la elaboración de modelos de situaciones próximas al contexto real del estudiante (ARTIGUE, 1995; BADILLO, 2003; MORENO, 2005; GARCÍA, 2013; ROJAS; GUACANEME, 2013; SÁNCHEZ-MATAMOROS; GARCÍA; LLINARES, 2008).

Se destaca que durante la consulta del estado del arte, se observó que existen diversos estudios focalizados a indagar, explorar y caracterizar las actitudes de los estudiantes hacia la Estadística y hacia la Matemática en general; no obstante, en el contexto mexicano se identificaron escasos registros de investigaciones sobre las actitudes hacia el cálculo diferencial e integral.

Se reconoce el valor del constructo actitud como uno de los componentes importantes de la subjetividad del ser humano, el cual se manifiesta en su modo de ser, de sentir y de actuar. Por tanto, se consideró pertinente realizar esta investigación con la intención de aportar conocimientos que motiven la discusión, el análisis y la comprensión del impacto de las actitudes de los estudiantes en su desempeño en el aula. 
Cabe señalar que este trabajo forma parte de una investigación más amplia sobre las actitudes y expectativas de estudiantes de bachillerato hacia el Cálculo como disciplina epistemológica, pero también hacia diversos aspectos que involucran una clase; por ejemplo, la metodología implementada por el profesor, las actividades de aula, los métodos de evaluación, la actitud del maestro en el aula, entre otros aspectos.

\section{Problematización}

La trayectoria profesional de los autores en diversos niveles de educación ha permitido vivenciar distintas situaciones escolares que han generado algunas preocupaciones. Entre las más recurrentes están el elevado número de estudiantes reprobados en la disciplina de Matemáticas al final de cada ciclo escolar y la falta de disposición e interés de estudiantes hacia el aprendizaje de esta disciplina.

Tales situaciones en el trascurrir del ejercicio docente ha motivado a reflexionar sobre: ¿por qué, históricamente, algunos estudiantes consideran las matemáticas una materia difícil de entender?, ¿por qué la implementación de algunas estrategias didácticas no logran despertar el interés de aprender matemáticas en el estudiante?, ¿por qué algunas metodologías de enseñanza no logran cambios actitudinales favorables en algunos estudiantes frente a la asignatura?, ¿por qué en el proceso de enseñanza y aprendizaje de las matemáticas, en ocasiones, se subvalora la subjetividad del estudiante?, ¿qué actitudes hacia las matemáticas caracterizan a los estudiantes y de qué manera éstas se relacionan y/o determinan su desenvolvimiento escolar?

En este artículo no se pretende dar respuestas a las anteriores preguntas. No obstante, esas interrogaciones permitieron reflexionar y tomar consciencia de la complejidad que envuelve el proceso de enseñanza y aprendizaje de las matemáticas y de los distintos matices que ella asume. Por lo tanto, indagar el aspecto actitudinal del estudiante en relación con lo que piensa, opina, siente y qué reacciones comportamentales le genera la clase de Matemáticas-Cálculo, proporciona valiosos conocimientos que auxilian la comprensión de distintas dimensiones que adopta el fenómeno de la reprobación escolar en esta materia.

Ahora bien, considerando que la actitud es un constructo que implica valores, creencias, conocimientos, opiniones, sentimientos y comportamientos (MANN, 1981), tal como se ampliará en una sección más adelante; en este estudio se planteó la siguiente pregunta de investigación: ¿qué actitudes hacia el cálculo diferencial e integral caracterizan a estudiantes de la Escuela de Bachilleres Salvador Allende Plantel Norte, de la Universidad 
Autónoma de Querétaro? Asimismo, con el propósito de responder esa interrogante se estableció como objetivo identificar, analizar y caracterizar las actitudes de los estudiantes hacia el curso de Cálculo diferencial e integral.

Con respecto al estado del arte, son diversos los estudios sobre la actitud hacia las matemáticas en el bachillerato que anteceden a esta investigación. Inicialmente, presentamos el estudio de Eudave (1994) quien centró su interés en las actitudes hacia las matemáticas de 541 estudiantes de la escuela de bachillerato propedéutico de la ciudad de Aguascalientes. De acuerdo con sus resultados, el 65,7\% de los alumnos presentó actitudes favorables hacia las matemáticas, un 31,2\% se mantuvo indeciso y un 3,1\% manifestó actitudes desfavorables.

Álvarez y Castañeda (2004), en su estudio realizado con trescientos estudiantes de los planteles Lic. Adolfo López Mateos y Nezahualcóyotl de la Escuela Preparatoria de la Universidad Autónoma del Estado de México, los resultados mostraron que en el primer plantel la mayoría de los alumnos manifestaron actitudes positivas hacia las matemáticas. Una menor proporción de alumnos opinó neutralmente y una parte mínima se manifestó de manera negativa. En el segundo plantel la mayoría de los alumnos manifestaron actitudes neutrales hacia las matemáticas; una menor proporción opinó negativamente y una parte mínima se manifestó positivamente hacia las matemáticas. Los autores demostraron la existencia de una relación entre la reprobación en matemáticas y la actitud de los estudiantes hacia esta materia.

Otras investigaciones (MARTÍNEZ, 2008, 2013; MATO; DE LA TORRE, 2010; MA; KISHOR, 1999, citados en JUÁREZ, 2010; MAAß; SCHLÖGLMANN, 2009; GIL; BLANCO; GUERRERO, 2005; HIDALGO; MAROTO; PALACIOS, 2004; GÓMEZCHACÓN, 2003; MCLEOD, 1988; FERNÁNDEZ, 1986) mostraron la relación existente entre la actitud, el aprendizaje y rendimiento escolar del estudiante en Matemáticas.

Por su parte, Gairín (1990) realizó un estudio en España sobre las actitudes en la educación en general, e hizo una particular referencia a las actitudes en la enseñanza y aprendizaje de las matemáticas. Mostró la importancia didáctica de las actitudes y, además, realizó una caracterización actitudinal en términos generales del estudiante en el aula. Se considera que los anteriores estudios son un referente de apoyo para identificar y comprender algunas manifestaciones actitudinales del estudiante en la clase de matemáticas.

\subsection{El concepto de Actitud}

Este estudio se fundamenta en la Teoría de las Actitudes, la cual se inscribe en el campo de la Psicología Social. Ahora bien, ¿qué es la actitud? Según Villoro (2002), este 
concepto ha desempeñado un papel central en las últimas décadas, y sin su ayuda no se hubieran desarrollado varios campos de la investigación empírica, lo cual basta para demostrar su operatividad - validez - científica.

Históricamente, en el desarrollo de la Psicología Social han surgido definiciones variadas sobre la actitud, no siempre compatibles entre sí. Y se perciben elementos comunes y diferenciadores de este constructo (MORALES; MOYA, 1996). El concepto de actitud denota la suma total de inclinaciones y sentimientos humanos, prejuicios o distorsiones, nociones preconcebidas, ideas, temores y convicciones acerca de un asunto determinado (THURSTONE, 1928, citado en SUMMERS, 1978).

Las actitudes tienen un significado adaptativo, puesto que representan un eslabón psicológico fundamental entre las capacidades de percibir, de sentir y de emprender de una persona, al mismo tiempo que ordenan y dan significación a su experiencia continua en un medio social complejo (KRECH; CRUTCHFIELD; BALLACHEY, 1962, citado en MANN, 1981). Asimismo, la actitud es la disposición fundamental que interviene junto con otras influencias en la determinación de una diversidad de conductas hacia un objeto en cuestión, la cual incluye declaraciones de creencias, sentimientos y acciones de aproximación o evitación con respecto a dicho objeto (COOK; SELTIZ, 1964, citado en SUMMERS, 1978).

Desde la perspectiva de Auzmendi (1992) las actitudes son aspectos no directamente observables sino inferidos, compuestos tanto por las creencias como por los sentimientos y las predisposiciones comportamentales hacia el objeto al que se dirigen. En ese mismo sentido, Morales y Moya (1996) consideran que la actitud se trata de una evaluación del objeto actitudinal y lleva siempre aparejada una comparación del valor del objeto con otro objeto.

Las creencias, los sentimientos y las conductas constituyen las formas en que la actitud se genera y se vivencia. Ahora bien, para los propósitos de esta investigación entendemos por actitud a la predisposición del estudiante a responder de manera positiva, indiferente o negativa hacia un objeto, es decir, su postura de rechazo, de indiferencia o aceptación hacia el objeto de actitud. En este estudio el objeto de actitud es el cálculo diferencial e integral.

Algunos autores han propuesto un modelo que define la actitud en tres componentes. De acuerdo con Mann (1981), en la actitud podemos distinguir el componente cognoscitivo, que refiere a percepciones, creencias, estereotipos, ideas etc., sobre el objeto. El afectivo, que se expresa a través de los sentimientos de una persona con respecto al objeto, y el componente comportamental, que se manifiesta en la tendencia a actuar o de reaccionar de un cierto modo con respecto al objeto, es decir, es la orientación a la acción. 
De igual forma Rosenberg y Hovland (1960, citado en MORALES; MOYA, 1996) establecieron tres elementos interrelacionados en la actitud. Primero, el cognitivo, el cual incluye el dominio de hechos, opiniones, creencias - especialmente de carácter evaluativo -, pensamientos, valores, y conocimientos relevantes acerca de un objeto. Segundo, el evaluativo, que corresponde a los sentimientos asociados a dicho objeto; son los procesos que avalan o contradicen las bases de nuestras creencias, expresados en sentimientos evaluativos, preferencias, estados de ánimo y emociones que se evidencian ante el objeto. Y, finalmente, el conductual que concierne a las intenciones de comportarse hacia ese objeto de una manera determinada; muestra las evidencias de actuación a favor o en contra del objeto de actitud.

A partir de los anteriores aportes teóricos sobre los componentes de la actitud, para efecto de la realización de este estudio, se define: a) Componente cognitivo: refiere a estados internos no observables pero inferidas a través de las enunciaciones verbales expresadas por el estudiante, en la que se incluyen, concepciones, creencias, opiniones acerca del Cálculo. Así como percepciones sobre la metodología de enseñanza, las actividades de aprendizaje, el método de evaluación y la autopercepción como aprendiz del Cálculo; b) Componente afectivo: refiere a estados de ánimo, emociones y sentimientos que el estudiante manifiesta ante el Cálculo como disciplina epistemológica y hacia la clase de Cálculo. Incluye sentimientos evaluativos, es decir, de tipo positivo, de indiferencia o negativo con respecto a las formas de enseñanza, a las actividades de aprendizaje, a las formas de evaluación del aprendizaje, entre otros aspectos relacionados con la clase; c) Componente conductual: refiere a las reacciones comportamentales manifestadas por el estudiante en la clase de Cálculo.

Aunado a lo anterior, se destaca que en el campo de la Educación Matemática, Gómez-Chacón (2009) distingue dos categorías en relación a las actitudes que se pueden manifestar hacia las Matemáticas. La primera es, Actitudes hacia las Matemáticas, la cual se manifiesta en términos de satisfacción, gusto, curiosidad, valoración, aprecio, alegría, miedo, desprecio, interés por esta disciplina y por su aprendizaje, entre otras. Subraya más el componente afectivo que el cognitivo. La segunda categoría es, Actitudes matemáticas. A diferencia de la primera categoría, ésta tiene un carácter exclusivamente cognitivo y se refiere al modo de utilizar las capacidades generales como la flexibilidad de pensamiento, la apertura mental, el espíritu crítico, la objetividad, el razonamiento, etc., en la actividad matemática.

A partir de lo anteriormente expuesto, nuestro interés de estudio se dirige a las Actitudes hacia las Matemáticas-Cálculo, delimitado en dos subcategorías, 1) Actitud hacia el Cálculo como disciplina curricular: imagen social del Cálculo y usos sociales del Cálculo valor, utilidad y/o aplicabilidad -; y 2) Actitud hacia la clase de Cálculo: actitud hacia la 
metodología de enseñanza, percepción sobre las actividades de aprendizaje y percepción sobre el método de evaluación.

\section{Procedimientos metodológicos}

En este estudio se optó por un método mixto. En el proceso de recolección de datos e información se utilizaron técnicas tanto de carácter cualitativo como de tipo cuantitativo. Asimismo, el estudio tuvo un diseño no experimental, transeccional y descriptivo. Esto es, la recolección de los datos se llevó a cabo en un sólo momento, espacio de tiempo y sin manipulación de variables. Con respecto a los estudios descriptivos, Cortés (2012) plantea que con ellos se busca especificar las propiedades importantes de las personas, grupos o comunidades o cualquier otro fenómeno que sea sometido al análisis, y en el cual se seleccionan varias cuestiones y se mide cada una de ellas independientemente para así decir cómo es y cómo se manifiesta el fenómeno de interés.

\subsection{Participantes}

La población estuvo conformada por 740 estudiantes de educación escolarizada, pertenecientes al semestre V de preparatoria de la Escuela de Bachilleres Salvador Allende Plantel Norte, de la Universidad Autónoma de Querétaro en la ciudad de Querétaro, México. Estos estudiantes cursaban la disciplina de Cálculo Diferencial e Integral. Asimismo, esta población estaba distribuida en veinte grupos; diez en el turno matutino y diez en el turno vespertino con un promedio de 37 estudiantes en cada salón de clases. La distribución de la población se observa en la Tabla 1.

Tabla 1- Distribución de la población de estudiantes por género y por turno escolar

\begin{tabular}{cccccc}
\hline \multirow{2}{*}{ Género } & \multicolumn{2}{c}{ Turno matutino } & \multicolumn{2}{c}{ Turno vespertino } & Total \\
\cline { 2 - 6 } & Participantes & $\%$ & Participantes & $\%$ & \\
Hombres & 171 & 23 & 144 & 20 & 315 \\
Mujeres & 240 & 32 & 185 & 25 & 425 \\
Total & 411 & 55 & 329 & 45 & 740 \\
\hline
\end{tabular}

Fuente: elaboración propia basada en investigación documental

De esa población se seleccionó una muestra del 20\%, correspondiente a 149 estudiantes cuyas edades oscilaron entre los 17 y 21 años (Tabla 2). Para asegurar la representatividad de la muestra se aplicó un muestreo probabilístico aleatorio simple, en el cual se seleccionó a los sujetos por medio de una tabla de números aleatorios. Un aspecto adicional que se tuvo en cuenta con respecto a la representatividad de la muestra fue 
conservar igual número porcentual de estudiantes tanto por turnos - matutino y vespertino como por sexo - mujer y hombre - con respecto al número porcentual obtenido en la población.

Tabla 2 - Distribución de la muestra de estudiantes por género y por turno escolar

\begin{tabular}{cccccc}
\multirow{2}{*}{ Género } & \multicolumn{2}{c}{ Turno matutino } & \multicolumn{2}{c}{ Turno vespertino } & Total \\
\cline { 2 - 6 } & Participantes & $\%$ & Participantes & $\%$ & 65 \\
Hombres & 35 & 23 & 30 & 20 & 84 \\
Mujeres & 48 & 32 & 36 & 25 & 149 \\
Total & 83 & 55 & 66 & 45 & \\
\hline
\end{tabular}

Fuente: elaboración propia basada en investigación documental

En el proceso de selección de la muestra se consideraron los siguientes criterios, a) de inclusión: incluir a aquellos estudiantes que de manera voluntaria se ofrecieran a participar en la entrevista y en el cuestionario; b) de exclusión: excluir a aquellos estudiantes que no quisieran participar en la aplicación de los instrumentos de recolección mencionados en el inciso anterior; c) de eliminación: eliminar o no considerar para efecto de la sistematización de la información obtenida a aquellos estudiantes que no respondieran más del $80 \%$ de los instrumentos.

A los 149 estudiantes correspondientes al tamaño muestral se les aplicó el cuestionario, en tanto que la entrevista fue realizada a veintiocho de ellos quienes aceptaron de manera voluntaria participar en la misma. Es importante destacar que, al momento de aplicar los instrumentos, los estudiantes se encontraban en la última semana del curso de Cálculo Diferencial e Integral. Este hecho fue significativo para el estudio debido a que los estudiantes tenían un conocimiento experiencial y sensible sobre el curso de Cálculo, lo cual generó en ellos una mayor seguridad para expresar con propiedad sus opiniones, sensaciones, sentimientos y percepciones sobre distintas situaciones y/o aspectos vivenciados en el Curso.

\subsection{Instrumentos}

En este estudio se utilizaron como técnicas de recolección de datos un cuestionario con formato Likert sobre actitudes hacia el cálculo diferencial e integral y una entrevista semiestructurada. Esas técnicas no se excluyeron ni se sustituyeron entre sí, sino por el contrario se complementaron en la búsqueda de aquellos atributos que caracterizaron las actitudes de los estudiantes hacia el Cálculo; lo cual permitió una amplia producción, exploración, riqueza y variedad de datos, así como una mejor comprensión del fenómeno estudiado (HERNÁNDEZ; FERNÁNDEZ; BAPTISTA, 2010). 
Un cuestionario con formato Likert es aquel que presenta una serie de ítems o enunciados los cuales tienen distintas alternativas de respuestas fijas para cada ítem. Generalmente las cinco alternativas de respuestas que se establecen son: Totalmente de acuerdo, De acuerdo, Neutral, En desacuerdo y Totalmente en desacuerdo. El formato Likert es conocido como una escala aditiva con un nivel nominal cuyo objetivo es medir la actitud que manifieste una persona con respecto a un objeto o situación.

Con respecto al cuestionario utilizado en el estudio, inicialmente se realizó un piloteo con un grupo de 76 estudiantes y, posteriormente, se sometió a un análisis de confiabilidad del Alfa de Cronbach en el que se obtuvo un resultado de 0.807; el cual se consideró apropiado. El cuestionario estuvo conformado por diecisiete ítems adaptados de la Escala de Actitudes hacia la Estadística de Auzmendi (1992) y de la Escala de Actitudes hacia la Estadística de Estrada (2011). Del total de ítems quince fueron formulados en un sentido positivo (ítems: $1,2,3,5,6,7,8,9,10,11,12,13,14,16,17$ ) y dos en sentido negativo (ítems: 4, 15 ).

Para facilitar el proceso de análisis de los datos recabados con este instrumento, se organizaron los diecisiete ítems en subcategorías y éstos a su vez se agruparon en tres componentes conforme a la teoría de las Actitudes (Cuadro 1).

\begin{tabular}{l} 
Componente cognitivo \\
\hline \multicolumn{1}{|c|}{ Cariable: Actitud } \\
Subcategoría 1: concepciones y opiniones \\
Ítem 1: considero el Cálculo como una materia muy necesaria en el bachillerato. \\
Subcategoría 2: valor y/o utilidad \\
Ítem 4: utilizo poco el Cálculo fuera de la escuela. \\
Ítem 8: saber utilizar el Cálculo incrementaría mis posibilidades de ingresar a la universidad. \\
Ítem 14: en mi futura carrera profesional una de las materias más importantes que ha de estudiarse es el \\
Cálculo. \\
Subcategoría 3: autopercepción \\
Ítem 6: tengo confianza en mí mismo (a) cuando me enfrento a un ejercicio o problema de Cálculo. \\
Ítem 16: si me lo propusiera creo que llegaría a dominar bien el Cálculo. \\
\hline \\
Subcategoría 1: emociones y/o sentimientos \\
Ítem 2: el estudiar o el trabajar con el Cálculo no me asusta en lo absoluto. \\
Ítem 3: el utilizar el Cálculo es una diversión para mí. \\
Ítem 7: me divierte hablar con otros sobre Cálculo. \\
Ítem 10: el Cálculo es agradable y motivante para mí. \\
Ítem 11: no me altero cuando tengo que trabajar en ejercicios o problemas de Cálculo. \\
Ítem 13: me provoca una gran satisfacción el llegar a resolver ejercicios o problemas de Cálculo. \\
Ítem 15: el Cálculo hace que me sienta incómodo (a) y ansioso (a). \\
Subcategoría 2: preferencias y/o gustos \\
Ítem 5: quiero llegar a tener un conocimiento más profundo del Cálculo. \\
Ítem 12: me gustaría tener una ocupación en la cual tuviera que utilizar el Cálculo. \\
Ítem 17: si tuviera oportunidad me inscribiría en más cursos de Cálculo de los que son necesarios. \\
\hline \\
Subcategoría 1: comportamiento y sentimiento \\
Ítem 9: estoy calmado(a), tranquilo(a) cuando me enfrento a un ejercicio o problema de Cálculo. \\
\hline
\end{tabular}

Cuadro 1- Distribución de los ítems del cuestionario con base en los componentes de la Actitud Fuente: elaboración propia con base en la teoría de las Actitudes y adaptación 
de las Escalas de Auzmendi (1992) y de Estrada (2011).

Con respecto a la entrevista, se elaboró una guía para la misma, la cual fue sometida a una validación de constructo y a un pilotaje previo a su aplicación. Luego de ese proceso, fue aplicada de manera individual a veintiocho estudiantes con una duración en promedio de cinco minutos por estudiante. Su finalidad fue la obtención de las enunciaciones discursivas de los estudiantes en relación a sus sentimientos, gustos, expectativas, concepciones acerca de la utilidad del cálculo, percepciones sobre la formas de evaluación y reacciones comportamentales en el aula. Las características de la guía de entrevista puede observarse en el Cuadro 2.

\section{Variable: Actitud}

1. Componente cognitivo: concepciones y opiniones

1.1. De lo que viste o aprendiste en tu curso, ¿para qué crees que sirve el Cálculo? Propósito: conocer la utilidad general que el estudiante le da a los conocimientos vistos y/o adquiridos en el Curso.

1.2. ¿Crees que los temas que viste en este semestre en Cálculo te han servido y, te servirán para algo? Propósito: reconocer la utilidad o importancia presente y futura de los temas abordados en el curso de Cálculo para la vida práctica o cotidiana del estudiante.

\section{Componente afectivo: sentimientos, gustos y/o preferencias}

2.1. ¿Cómo te sientes en la clase de Cálculo? Propósito: identificar manifestaciones de sentimientos o emociones hacia la clase de Cálculo.

2.2. ¿Cómo te gustaría que fuese la clase de Cálculo? Propósito: indagar características que los estudiantes le atribuyen a una clase de Cálculo de su agrado.

2.3. ¿Cómo te evalúan en la clase de Cálculo?, ¿Te gusta la forma en que te evalúan? Propósito: indagar sobre los métodos y/o estrategias utilizados para evaluar el desempeño de los estudiantes y conocer su nivel de agrado con respecto a ellos.

\section{Componente conductual: acciones o comportamientos}

3.1. ¿Cómo te comportas o reaccionas en la clase de Cálculo? Propósito: identificar tipos de comportamientos o actuaciones del estudiante en el aula.

Cuadro 2 - Características y propósitos de la guía de entrevista

Fuente: elaboración propia con base en la teoría de las Actitudes

\section{Resultados}

En este apartado se presentan los resultados del cuestionario y de la entrevista. Con respecto a los datos recabados por medio del cuestionario, éstos fueron sistematizados con el programa Statistical Package for the Social Sciences (SPSS) versión 14. Los ítems se analizaron uno por uno en el que se obtuvieron las tendencias porcentuales a través de estadística descriptiva.

En ese proceso de análisis se identificaron sentidos tanto positivos como negativos de la actitud de los estudiantes hacia los distintos tópicos consultados relacionados con el cálculo. Con respecto al proceso de análisis de la entrevista se optó por un Análisis de Contenido (AC), en la cual se identificaron las unidades de significado y se organizaron en categorías de acuerdo con los tres componentes de la actitud según la teoría de apoyo. Para 
Bardin (2009) el AC consiste en un conjunto de técnicas de análisis de las comunicaciones y procedimientos sistemáticos y objetivos de descripción del contenido de los mensajes cuantitativos, cualitativos, otros - que permiten la inferencia de conocimientos relativos a las condiciones de producción/recepción de esos mensajes.

\subsection{Del cuestionario de actitudes}

En este apartado se presentan los resultados generales obtenidos en el cuestionario lo cual permitió identificar posturas de rechazo, de indiferencia y de aceptación de los estudiantes hacia determinados aspectos vinculados con la disciplina de cálculo diferencial e integral.

Para efectos de mostrar las tendencias actitudinales de los estudiantes hacia el objeto de estudio en cuestión y facilitar el proceso de análisis, se decidió presentar los resultados de tal forma que se integró porcentualmente algunas alternativas de respuestas, en la cual se establecieron finalmente tres alternativas generales, como se indica a seguir: 1) De acuerdo, corresponde a la sumatoria porcentual de las opciones de respuestas Totalmente de acuerdo y De acuerdo, 2) En desacuerdo, corresponde a la sumatoria porcentual de las opciones de respuestas Totalmente en desacuerdo y En desacuerdo, y por último, 3) Neutral.

Asimismo, los resultados son presentados en subcategorías reagrupadas en tres categorías generales de análisis correspondientes a los componentes de la actitud, como describiremos a continuación (Cuadro 3).

Subcategoría 1: concepciones y opinión

\section{Componente cognitivo}

Ítem 1: considero el Cálculo como una materia muy necesaria en el bachillerato. De acuerdo $(69,8 \%)$; En desacuerdo $(10,7 \%)$ y Neutral $(19,5 \%)$.

Subcategoría 2: valor y/o utilidad

Ítem 4: utilizo poco el Cálculo fuera de la escuela. De acuerdo (53\%); En desacuerdo (20,2\%) y Neutral $(26,8 \%)$.

Ítem 8: saber utilizar el Cálculo incrementaría mis posibilidades de ingresar a la universidad. De acuerdo $(78,6 \%)$; En desacuerdo $(8,7 \%)$ y Neutral $(12,7 \%)$.

Ítem 14: en mi futura carrera profesional una de las materias más importantes que ha de estudiarse es el Cálculo. De acuerdo (30,9\%); En desacuerdo (48,3\%) y Neutral $(20,8 \%)$.

Subcategoría 3: autopercepción

Ítem 6: tengo confianza en mí mismo(a) cuando me enfrento a un ejercicio o problema de Cálculo. De acuerdo (42\%); En desacuerdo (33\%) y Neutral (25\%).

Ítem 16: si me lo propusiera, creo que llegaría a dominar bien el Cálculo. De acuerdo $(79,8 \%)$; En desacuerdo $(6,7 \%)$ y Neutral $(13,5 \%)$.

Subcategoría 1: sentimientos y/o emociones

\section{Componente afectivo}

Ítem 2: el estudiar o el trabajar con el Cálculo no me asusta en lo absoluto. De acuerdo (42,3\%); En desacuerdo $(28,1 \%)$ y Neutral $(29,5 \%)$.

Ítem 3: el utilizar el Cálculo es una diversión para mí. De acuerdo (15\%); En desacuerdo (57\%) y Neutral $(27 \%)$. 
Ítem 7: me divierte hablar con otros sobre Cálculo. De acuerdo (12\%); En desacuerdo (69\%) y Neutral $(19 \%)$.

Ítem 10: el Cálculo es agradable y motivante para mí. De acuerdo (18\%); En desacuerdo (51\%) y Neutral $(31 \%)$.

Ítem 11: no me altero cuando tengo que trabajar en ejercicios o problemas de Cálculo. De acuerdo (30\%); En desacuerdo (33\%) y Neutral (37\%).

Ítem 13: me provoca una gran satisfacción el llegar a resolver ejercicios o problemas de Cálculo. De acuerdo (58\%); En desacuerdo (28\%) y Neutral (14\%).

Ítem 15: el Cálculo hace que me sienta incómodo(a) y ansioso(a). De acuerdo (34\%); En desacuerdo $(30 \%)$ y Neutral $(36 \%)$.

Subcategoría 2: preferencias y/o gustos

Ítem 5: quiero llegar a tener un conocimiento más profundo del Cálculo. De acuerdo (44\%); En desacuerdo (26\%) y Neutral (30\%).

Ítem 12: me gustaría tener una ocupación en la cual tuviera que utilizar el Cálculo. De acuerdo (19\%); En desacuerdo $(62 \%)$ y Neutral $(19 \%)$.

Ítem 17: si tuviera oportunidad me inscribiría en más cursos de Cálculo de los que son necesario. De acuerdo (42\%); En desacuerdo (31\%) y Neutral (27\%).

Subcategoría 1: reacción comportamental

\section{Componente conductual}

Ítem 9: estoy calmado(a), tranquilo(a) cuando me enfrento a un ejercicio o problema de Cálculo. De acuerdo (31\%); En desacuerdo (38\%) y Neutral (31\%).

Cuadro 3 - Resultados del cuestionario sobre actitudes hacia el Cálculo

Fuente: elaboración propia con base en los resultados del cuestionario sobre actitudes

A partir del cuadro anterior se evidencian distintas tendencias y/o posturas actitudinales de los estudiantes frente a diferentes cuestiones vinculadas al curso de Cálculo Diferencial e Integral. Con respecto al componente cognitivo, se percibe que la mayor parte de los estudiantes reafirmaron su convicción de que el Cálculo es una materia importante del currículo escolar y poseer conocimientos sobre esa disciplina incrementa sus posibilidades de ingreso a la universidad. Pero, también reconocieron que una de las debilidades del Curso es el poco uso y/o utilidad de estos conocimientos adquiridos en la vida extraescolar.

No obstante, un grupo de estudiantes $(30,9 \%)$ resaltó que en la futura carrera profesional a la que aspiran estudiar, una de las materias importantes a cursar es el cálculo. Por otro lado, un número destacado de estudiantes aseveró que si se propusieran como meta dominar el cálculo, lo lograrían. Sin embargo, sólo la mitad de este grupo de estudiantes aseguró tener confianza en ellos mismos al enfrentarse a un ejercicio o situación problema que involucre dichos conocimientos disciplinarios.

A lo que respecta a la autopercepción, menos de la mitad de los consultados manifestó tener confianza en sí mismos al enfrentarse a un ejercicio o problema. El grupo restante (58\%) se ubicó en una postura entre contraria y neutral.

Un porcentaje significativo de estudiantes $(69 \%)$ manifestó, con contundencia, que hablar con otros compañeros sobre cálculo no les resultó divertido. Este hecho podría explicar que el (44\%) de ellos expresaran su deseo de llegar a tener un conocimiento más profundo del cálculo frente a los demás que se mostraron en contra e indiferentes frente a ese deseo. 
También se evidencia que más de la mitad de los estudiantes (62\%) encuestados no desearían una ocupación en donde tuvieran que utilizar el Cálculo. Tanto así que una tercera parte de ellos expresó sentimientos de alteración e incomodidad al momento de trabajar en ejercicios o situaciones problema relacionados con esta disciplina. Se infiere que podría existir una relación entre esos contrastes, en el entendido de que a mayor dificultad reconocida por los estudiantes ante la disciplina, existe consecuentemente, un mayor deseo de superar sus dificultades/carencias conceptuales.

Esta situación se corrobora al observar que el $42 \%$ de los estudiantes expresó que si tuvieran la oportunidad, se inscribirían en más cursos de cálculo de los que son necesarios, todo ello con el propósito de desarrollar y/o potenciar habilidades matemáticas. Se destaca que en este componente afectivo, los estudiantes presentan una mayor tendencia hacia lo desfavorable o negativo con respecto al cálculo.

Finalmente, en relación con las reacciones comportamentales, sólo una tercera parte de los estudiantes manifestó asumir una actitud de tranquilidad y calma al momento de enfrentarse a resolver ejercicios o situaciones problema; en contraste con el resto de estudiantes (69\%) quienes adoptaron una postura actitudinal neutral y, además, se mostraron ansiosos en la clase de cálculo.

\subsection{De la entrevista sobre actitudes}

En este apartado se exhiben los resultados más sobresalientes de la entrevista correspondientes a cada uno de los componentes de la actitud, a saber, lo cognitivo, lo afectivo y lo conductual; lo que permite hacer una configuración actitudinal de los estudiantes investigados con relación al cálculo a partir de sus discursos proferidos.

\subsubsection{Componente cognitivo}

Con respecto al uso o aplicación de los conocimientos adquiridos sobre el cálculo diferencial e integral, aproximadamente la mitad (46\%) de los estudiantes manifestaron que se encuentra en el mismo campo de las matemáticas. Es decir, lo aprendido en el Curso fue utilizado para la realización de ejercicios de aplicación de algoritmos - límites, derivadas e integrales, resolución de situaciones problema, estímulo al razonamiento y a la agilidad mental, aplicación en situaciones estadísticas - y repaso o recordatorio de las matemáticas vistas en cursos anteriores - Álgebra, Geometría y Trigonometría. 
Un 35\% de ellos manifestó otros usos o aplicaciones del cálculo, como por ejemplo, en el área empresarial, industrial, comercial y tecnológica. Un grupo menor consideró que el cálculo sirve para quienes tienen pensado hacer estudios de licenciatura relacionados con las ingenierías, contaduría y administración (14\%), y una minoría expresó desconocer su utilidad $(5 \%)$.

En cuanto al uso del cálculo a nivel personal, una proporción elevada de los estudiantes (71\%) manifestó que sí les ha sido útil, en contraste con un 29\%. No obstante, la utilidad personal manifestada por ese primer grupo de estudiantes estuvo ligada a varios aspectos, a saber: percibieron el cálculo como la disciplina más importante del semestre V, y manifestaron que los conocimientos y habilidades adquiridas en el curso han facilitado la comprensión de otros contenidos curriculares abordados en otras disciplinas - Estadística, Física, Química, Economía.

En relación con la opinión de los estudiantes sobre la utilidad futura de los conocimientos adquiridos sobre el Cálculo, el $57 \%$ expresó que serían útiles, mientras que el $39 \%$ indicó lo contrario. Es importante referir, en ese ítem, que en relación a la utilidad futura de los conocimientos de Cálculo, algunos estudiantes respondieron en virtud de la formación profesional o universitaria a la que aspiraban, como por ejemplo, ingeniería, contabilidad, administración, entre otras; mientras que otros asociaron la utilidad futura de esos conocimientos con actividades de tipo comercial especialmente en el contexto de compraventas.

Llama la atención que este último grupo de estudiantes vincula la utilidad de los conocimientos de cálculo diferencial e integral específicamente con operaciones básicas de tipo cálculo aritmético y estadístico.

\subsubsection{Componente afectivo}

Con respecto a la afectividad de los estudiantes hacia el cálculo, se observó una variedad de emociones y sentimientos tanto positivos como negativos, siendo estos últimos los de mayor recurrencias (71\%): confusión, dificultad, aburrimiento, desinterés, distracción, desconcentración, nerviosismo, inquietud, estrés, frustración, fastidio, enojo, disgusto, ansiedad y baja autoestima. Con respecto a los sentimientos de tipo positivo, una tercera parte de los estudiantes (29\%) destacó: bienestar, agrado, seguridad y tranquilidad. Es de resaltar que esa tendencia afectiva desfavorable hacia el cálculo se asemeja a los resultados obtenidos en el cuestionario. 
Con relación a la pregunta, ¿cómo te gustaría que fuese la clase de Cálculo?, los atributos más sobresalientes expresados por los estudiantes fueron: didáctica y dinámica $(87 \%)$, con maestros estrictos y que se hagan respetar $(5,2 \%)$, sin favoritismo por parte del maestro $(2,6 \%)$, con maestros que no sean sarcásticos $(2,6 \%)$ y con maestros que se interesen y/o apoyen al estudiante en las actividades en el aula (2,6\%).

En cuanto a las formas de evaluar implementadas por los profesores, los resultados colocaron en evidencia que el método más recurrente para evaluar el desempeño de los estudiantes en la clase de Cálculo fue el examen escrito (74\%). En menor porcentaje se encontraron otras formas y/o criterios de evaluación como la participación en clase, los trabajos escritos y las tareas $(26 \%)$.

A lo que respecta al grado de satisfacción de los estudiantes hacia estos métodos de evaluación, la mayoría de ellos mostró su rechazo al predominio del examen escrito como el instrumento más recurrente por parte de los profesores para determinar sus avances en el aprendizaje de la disciplina (79\%); mientras que el restante manifestó sentirse a gusto con dicha exclusividad en la forma de evaluar (21\%).

\subsubsection{Componente conductual}

Con relación a las manifestaciones de comportamientos en la clase de cálculo, más de la mitad $(57 \%)$ de los estudiantes adoptaron una postura de atención en las explicaciones del profesor. En este aspecto es importante indicar que los estudiantes expresaron que el prestar atención a las explicaciones del maestro no significó que les interesara o gustara la clase, debido a que su interés principal era acreditar/aprobar la materia independientemente de las emociones negativas que ésta les generara, y en lo posible, evitar problemas interpersonales con el maestro.

En contraste, los demás estudiantes (36\%) adoptaron comportamientos negativos en el salón de clases, tales como desinterés, desconcentración, timidez, nerviosismo y parálisis o bloqueo mental al no entender la explicación de algunos temas. Por último, una minoría (4\%) de estudiantes adoptó un comportamiento de indiferencia hacia la clase debido a que no les generó ningún tipo de reacción, ni positiva ni negativa.

\section{Discusión}

Con base en los resultados del cuestionario y de la entrevista se constata que las 
posturas actitudinales de los estudiantes de la Escuela de Bachilleres Salvador Allende, Plantel Norte, hacia algunos aspectos vinculados con la clase de cálculo fueron variadas. Se observó que los estudiantes que atribuyeron una gran importancia a los conocimientos de cálculo percibían la disciplina como una herramienta para el desarrollo de capacidades cognitivas y un medio para la consecución de sus objetivos escolares y la aprobación del examen de ingreso a la universidad.

Las concepciones de los estudiantes sobre la aplicación del cálculo están encaminadas a concebirlo como un conjunto de conocimientos estáticos con aplicabilidad, principalmente, en el contexto propio de las matemáticas. De ahí, se infiere la existencia de una visión platónica de las matemáticas por parte de los estudiantes, es decir, una visión reduccionista de su aplicabilidad, en la cual la circunscriben, principalmente, al ámbito académico carente de un sentido social.

Este resultado es consistente con lo encontrado por Cubillo y Ortega (2000) y Eudave (1994) quienes afirmaron que la importancia de las matemáticas concedida por los estudiantes está relacionada a su utilidad en la formación universitaria. Tales resultados también se asemejan con los obtenidos por Gómez-Chacón (2009), quien reportó que los estudiantes consideraron a las matemáticas como una ciencia concluida y sin avances.

Por su parte, Ruiz et al. (2011) encontraron que los estudiantes les concedieron una utilidad a las matemáticas que no trasciende la clase, en tanto es considerada como un conjunto de métodos y procedimientos para resolver ejercicios y algoritmos. Algo semejante fue encontrado por Mejía (2010), quien destacó que los estudiantes percibieron que las matemáticas que aprenden en el salón de clases difícilmente les servirán en y para la vida cotidiana.

Esta situación es preocupante debido a que este tipo de creencias y concepciones influyen de manera significativa en la adopción de actitudes desfavorables hacia esta disciplina (RODRÍGUEZ, 2011); lo cual según D`Amore (2005) afecta, inclusive, toda la actividad del mismo maestro. No obstante, los argumentos expuestos anteriormente contrastan con el estudio de Eudave (1994), quien reportó que más de la mitad de los estudiantes de bachillerato presentó actitudes favorables hacia las matemáticas, manifestando que éstas son agradables, creativas e indispensables para la sociedad.

Parece ser que los sentimientos extremos que suscitan las Matemáticas es una de las características de esta disciplina del conocimiento. A este respecto Gairín (1990) destacó, en su estudio, que las matemáticas figuran como una de las materias más preferidas, pero al mismo tiempo la más rechazada para algunos. 
En referencia a la dimensión afectiva de la actitud, los estudiantes manifestaron diversos sentimientos, en los cuales los negativos fueron de mayor transcendencia. Estos sentimientos fueron motivados, primero, por la complejidad de la misma disciplina en algunos tópicos y, segundo, por el ambiente generado en el aula. Este hecho se asemeja a los resultados obtenidos por Martínez, G. (2011) en su estudio, quien indagó sobre las representaciones sociales que poseen estudiantes del nivel medio superior acerca del aprendizaje y enseñanza de las matemáticas en el contexto mexicano.

Los resultados de esta investigación ponen en evidencia que la metodología de enseñanza utilizada por el maestro fue un factor determinante en la actitud asumida por los estudiantes en la clase de cálculo. Los métodos implementados por el maestro se ubicaron en el principal aspecto de la clase que más generó desagrado, toda vez que los estudiantes expresaron su incomodidad y enojo por la forma rápida en que el maestro explicaba un tema, y la falta de estrategias didácticas para la enseñanza y aprendizaje del mismo.

Con respecto a esta situación, Díaz-Barriga y Hernández (2010) reafirman la pertinencia y la necesidad por parte del maestro de apropiarse de una diversidad de estrategias de enseñanza tendientes a estimular el aprendizaje significativo. Es de destacar que, en la actualidad, los maestros disponen de una variedad de estrategias y recursos centrados en el alumno y en su proceso de construcción de conceptos matemáticos, y algunos con la mediación de las tecnologías de la información y la comunicación que brindan nuevas formas de generar en el aula ambientes propicios para estimular el aprendizaje hacia las MatemáticasCálculo.

Con respecto a la actitud asumida por los estudiantes ante las formas de enseñanza del profesor, Shoenfeld (1992, citado en VILANOVA et al., 2005) afirma que las percepciones de estudiantes sobre las matemáticas es determinada o afectada, al menos en parte, por el maestro. Este mismo autor sostiene que la naturaleza del entorno que crea el maestro tiene una fuerte influencia en la manera en que los estudiantes conciben no sólo las matemáticas como ciencia, sino también las características que definen la actividad matemática.

Esta situación también fue reafirmada por Mejía (2010), en cuyo estudio la mayoría de los estudiantes percibió una actitud poco favorable del maestro mientras impartía la clase de matemáticas, lo cual no favoreció una relación de alteridad armoniosa entre discente y docente.

Es importante centrar la atención en lo relacionado con la actitud del maestro versus la actitud del estudiante, dada la afectación mutua que se presenta en su interacción en el aula. Para dar cuenta de esta afirmación se referencia el testimonio de algunos estudiantes que 
ejemplifican la manera en que la actitud adoptada por el maestro de cálculo se constituyó en detonador de ciertas actitudes negativas por parte de ellos con respecto a la clase. Este fenómeno se ilustra en el Cuadro 4.

\begin{tabular}{|c|c|c|}
\hline Acción del maestro & Reacción del estudiante & $\begin{array}{c}\text { Actitud adoptada por el } \\
\text { estudiante }\end{array}$ \\
\hline $\begin{array}{l}\text { Situación A: colocó ejercicios y } \\
\text { problemas en el pizarrón. Luego } \\
\text { se sentó en su escritorio a jugar } \\
\text { con su Ipod/celular durante toda } \\
\text { la clase y no atendió a las dudas e } \\
\text { inquietudes surgidas en el } \\
\text { estudiante. } \\
\text { Situación B: llegó al salón de } \\
\text { clases, entregó el taller a los } \\
\text { estudiantes y se sentó en su } \\
\text { escritorio. Cuando el estudiante } \\
\text { acudió a él para solicitar } \\
\text { explicación sobre } \\
\text { procedimiento en el que quedó } \\
\text { atorado, el maestro le respondió: } \\
\text { tú ya deberías saber eso, lo viste } \\
\text { en el semestre anterior. }\end{array}$ & $\begin{array}{l}\text { - Se puso a conversar con } \\
\text { algunos compañeros y no le } \\
\text { dio importancia a la clase. } \\
\text { - Se salió del salón de clase. } \\
\text { - Se puso a jugar y chatear con } \\
\text { su celular. } \\
\text { - No trabajó durante la clase. } \\
\text { - Guardó el taller y se puso a } \\
\text { adelantar tareas de otras } \\
\text { asignaturas. }\end{array}$ & $\begin{array}{l}\text { - Manifestó sentimientos de } \\
\text { frustración y molestia. } \\
\text { - Percibió la clase de Cálculo } \\
\text { aburrida y los talleres complicados } \\
\text { de resolver. } \\
\text { - Perdió el interés por el tema. } \\
\text { - Adoptó una percepción negativa } \\
\text { del profesor y de la clase de } \\
\text { cálculo. }\end{array}$ \\
\hline
\end{tabular}

\section{Cuadro 4 - Relación actitudinal entre maestro y estudiante en el aula} Fuente: elaboración propia basada en los testimonios de la entrevista

Evidentemente, en el proceso escolar, el maestro es uno de los agentes claves en la generación de actitudes tanto positivas como negativas en el estudiante hacia la disciplina que imparte. Por tanto, se torna un facilitador y estimulador tanto de actitudes favorables como desfavorables en el aula de Matemáticas. En ese sentido, se considera que el maestro puede constituirse en un actor fundamental en el proceso de transformación de actitudes negativas hacia actitudes positiva en el estudiante mediante la adopción de una actitud y una práctica docente que armonice con las expectativas, necesidades e intereses del estudiante.

En el contexto de la Educación Matemática, el estudio de los aspectos actitudinales del estudiante está tomando fuerza como una dimensión fundamental de su subjetividad aliada en la consecución del éxito escolar. En ese orden de ideas, Llinares (2003) establece una categorización de seis competencias básicas para identificar a un estudiante matemáticamente competente. Entre esas competencias propone la actitud positiva hacia las actividades o situaciones matemáticas.

Desde ese enfoque, es importante que el maestro movilice su potencial pedagógicodidáctico para promover y/o favorecer actitudes positivas hacia las Matemáticas, debido a que éstas, como entidades de la conducta humana no son estables, sino por el contrario, son susceptibles a ser modificadas a partir de la interacción social y/o experiencias de cada estudiante con la actividad matemática (JACKSON, 1981 citado en PÉREZ, 1996). 
Es importante que la práctica docente se caracterice por la criticidad y la reflexividad sobre los distintos fenómenos escolares asociados con la actitud del estudiante, de tal manera que brinde posibilidades de conocer, comprender e intervenir dichos fenómenos con la puesta en marcha de propuestas pedagógicas conducentes a minimizar ciertas complejidades con respecto al aprendizaje de las Matemáticas-Cálculo.

\section{Consideraciones finales}

Esta investigación, cuyo eje central fueron las actitudes hacia el cálculo diferencial e integral que caracterizaron a los estudiantes de la Escuela de Bachilleres Salvador Allende, Plantel Norte, de la ciudad de Querétaro, motiva a explicitar algunas consideraciones. Primeramente, este estudio permitió ampliar nuestro conocimiento sobre la relación entre las variables actitud y aprendizaje de las Matemáticas-Cálculo.

Adquirimos mayor consciencia de la importancia de valorar, como elemento importante del proceso escolar, el conocimiento de tipo subjetivo del estudiante el cual se manifiesta en sus percepciones, creencias, auto-conceptos, representaciones sociales, sentimientos, gustos, intereses, expectativas, reacciones comportamentales, tensiones y preocupaciones en relación a las matemáticas como contenido epistemológico - disciplina curricular - y hacia algunos aspectos que envuelven la clase - metodología de enseñanza, actividades de aprendizaje, formas de evaluación, actitud del profesor en clase, entre otros.

Es importante destacar que existen otras variables que se constituyen en factores generadores de actitudes particulares - positivas, negativas - de los estudiantes hacia el cálculo tales como la naturaleza de los conceptos - lenguaje simbólico, alto nivel de abstracción, procesos al infinito que representan un obstáculo epistemológico etc. - y la transposición didáctica llevada a cabo por el docente en el aula, lo que podría facilitar u obstaculizar la comprensión y apropiación/construcción de conceptos.

Teniendo en cuenta los resultados en relación a los estudiantes con una actitud negativa hacia el contenido de cálculo y hacia distintos aspectos ya mencionados vinculados con la clase, es motivo de preocupación observar que en el sistema escolar mexicano continúen egresando bachilleres con una percepción desfavorable hacia esta disciplina.

Esa realidad vivenciada en la Escuela de Bachilleres en la cual ingresa semestralmente el mayor número de estudiantes de la ciudad de Querétaro, genera como efecto colateral el egreso de bachilleres portadores y reproductores de una ideología sobre las MatemáticasCálculo, que a partir de sus experiencias no gratas con la disciplina, refuerza social y 
culturalmente imaginarios y estereotipos cargados de prejuicios sobre ella. Esta situación posibilita, aún más, la perpetuación histórica de las matemáticas como la disciplina más complicada y tediosa del plan de estudio del bachillerato.

Las reflexiones suscitadas en este estudio permiten sensibilizar y ampliar los conocimientos sobre las implicaciones de la relación actitud↔aprendizaje de las matemáticas en el ámbito escolar. Con base en los discursos emitidos por los estudiantes en la entrevista, destacamos que la actitud del estudiante es un factor determinante en el alcance o fracaso de los objetivos de aprendizajes propuestos en el currículo de Matemáticas. Por tanto, se sugiere que las actitudes de los estudiantes sean exploradas y tomadas en cuenta en la planeación escolar, con el fin de crear puentes y/o puntos de encuentro entre las expectativas, intereses e inquietudes del aprendiz con las expectativas curriculares, todo ello con la mediación dialógica en el aula entre maestro y estudiante.

Finalmente, consideramos que los estudios sobre las actitudes de estudiantes hacia las matemáticas se constituyen en aportes valiosos para: a) motivar cada vez más las discusión y la comprensión de la actitud como una variable que influencia de forma positiva y/o negativa el desenvolvimiento escolar del estudiante; b) proporcionar insumos teóricos y experienciales que contribuyan a mejorar y comprender las prácticas escolares en el marco de la enseñanza y aprendizaje de las matemáticas en México y en otros contextos internacionales.

\section{Referencias}

ÁLVAREZ, D.; COLORADO, H.; OSPINA, L. Una propuesta didáctica para la enseñanza del concepto de derivada. Revista Educación científica y tecnológica, Bogotá, Edición especial, p. 201205, 2013.

ÁLVAREZ, M.; CASTAÑEDA, A. La reprobación en Matemáticas. Dos experiencias. Tiempo de Educar, México, v. 5, n. 9, p. 141-172, 2004.

ARTIGUE, M. Enseñanza y aprendizaje del análisis elemental: ¿qué se puede aprender de las investigaciones didácticas y los cambios curriculares? RELIME, México, v. 1, n. 1, p. 40-55, 1998.

ARTIGUE, M. La enseñanza de los principios del cálculo: problemas epistemológicos, cognitivos y didácticos. En: GÓMEZ, P. (Ed.). Ingeniería didáctica en educación matemática: Un esquema para la investigación y la innovación en la enseñanza y el aprendizaje de las matemáticas. Bogotá: Grupo Editorial Iberoamericano, 1995. p. 97-140.

AUZMENDI, E. Las Actitudes hacia la Matemática-Estadística en las enseñanzas Medias y

Universitarias. Características y medición. 1ª ed. España: Mensajero, 1992.

BADILLO, E. La derivada como objeto matemático y como objeto de enseñanza y aprendizaje en profesores de matemática de Colombia: "la derivada un concepto a caballo entre la matemática y la física". 449 p. Tesis (Doctorado de Didáctica de las ciencias y de las Matemáticas) - Departamento 
de Didáctica de la Matemática y de las Ciencias Experimentales, Universidad Autónoma de Barcelona, Barcelona, España, 2003.

BARDIN, L. Análise de conteúdo. Reimpressão da Edição Revista e Actualizada. Lisboa: Edições 70, 2009.

CORTÉS, M. Metodología de la investigación. 1ª ed. México: Trillas, 2012.

CUBILLO, C.; ORTEGA, T. Influencia de un modelo didáctico en la opinión/actitud de los alumnos hacia las Matemáticas. RELIME, México, v. 3, n. 2, p. 189-206, 2000.

D`AMORE, B. Bases filosóficas, pedagógicas, epistemológicas y conceptuales de la didáctica de la matemática. $1^{\text {a }}$. ed. España: Reverté, 2005.

DÍAZ-BARRIGA, F.; HERNÁNDEZ, G. Estrategias docentes para un aprendizaje significativo. Una interpretación constructivista. $3^{\text {a }}$. ed. México: Mc Graw Hill, 2010.

ESTRADA, A. Instrumentos de medición de actitudes hacia la Estadística: la escala EAEE para profesores. En: MORENO, M.; CLIMENT, C. (Org.). Investigación en Educación Matemática. Comunicaciones de los Grupos de Investigación de la SEIEM, 2011. p. 233-251.

EUDAVE, D. Las actitudes hacia las matemáticas de los maestros y alumnos de bachillerato. Revista Educación Matemática, México, v. 6, n. 1, p. 47-58, 1994.

FERNANDEZ, M. Evaluación y cambio educativo: El fracaso escolar. $1^{\text {a }}$. ed. España: Morata, 1986.

GAIRÍN, J. Las actitudes en educación. Un estudio sobre educación matemática. $1^{a}$. ed. España: Boixareu Universitaria, 1990.

GARCÍA, J. La problemática de la enseñanza y el aprendizaje del cálculo para ingeniería. Revista Educación, Costa Rica, v. 37, n. 1, p. 29-42, 2013.

GIL, N.; BLANCO, L.; GUERRERO, E. El dominio afectivo en el aprendizaje de las Matemáticas. Una revisión de sus descriptores básicos. UNION Revista Iberoamericana de Educación, Brasil, n. 2. p. 15-32, 2005.

GÓMEZ-CHACÓN, I. Actitudes matemáticas: propuesta para la transición a la universidad. Revista Educación Matemática, México, v. 21, n. 3, p. 5-32, 2009.

GÓMEZ-CHACÓN, I. La tarea intelectual en matemáticas: afecto, meta-afecto y los sistemas de creencias. Boletín de la Asociación Matemática Venezolana, Venezuela, v. 10, n. 2, p. 225-247, 2003.

GONZÁLEZ, L.; RADILLO, M. Una propuesta para la enseñanza del concepto de derivada de una función, mediante actividades de visualización. Acta Latinoamericana de Matemática Educativa, México, n. 27, p. 925-932, 2014.

GÜICHAL, E. et al. V. La enseñanza del cálculo en la educación polimodal y en la universidad. Diagnóstico sobre números reales. Acta Latinoamericana de Matemática Educativa, México, n. 19, p. 303-309, 2005.

HERNÁNDEZ, R.; FERNÁNDEZ, C.; BAPTISTA, M. Metodología de la investigación. $5^{\mathrm{a}}$. ed. México: Mc Graw Hill, 2010. 
HIDALGO, S.; MAROTO, A.; PALACIOS, A. ¿Por qué se rechazan las matemáticas? Análisis evolutivo y multivariante de actitudes relevantes hacia las matemáticas. Revista de Educación, España, n. 334, p. 75-95, 2004.

IRAZOQUI, E.; MEDINA, A. Estudio preliminar de aproximación al concepto de límite de una función. Revista Theoria, Ciencia, Arte y Humanidades, Chile, v. 22, n. 1, p. 21-31, 2013.

JUÁREZ, J. Actitudes y rendimiento en Matemáticas. $1^{\text {a }}$. ed. México: Ediciones Díaz de Santos, 2010.

LLINARES, S. Matemáticas escolares y competencia matemática. En: Chamorro, M. (Org.).

Didáctica de las matemáticas para primaria. España: Pearson Prentice Hall, 2003. p. 3-30.

MAAß, J.; SCHLÖGLMANN, W. Beliefs and attitudes in Mathematics Education. New Research Results. $1^{\text {a }}$. ed. Países Bajos/China: Sense Publishers, 2009.

MANN, L. Elementos de psicología social. 8ª ed. México: Limusa, 1981.

MARTÍNEZ, G. Representaciones sociales que poseen estudiantes del nivel medio superior acerca del aprendizaje y enseñanza de las Matemáticas. Revista Perfiles Educativos, México, v. 33, n. 132, p. 90-109, 2011.

MARTÍNEZ, O. Actitudes hacia la matemática. Sapiens: Revista Universitaria de Investigación, Venezuela, v. 9, n. 1, p. 237-256, 2008.

MARTÍNEZ, O. Una mirada al fracaso en el aula de matemática a la luz del afecto. En: CONGRESO IBEROAMERICANO DE EDUCACIÓN MATEMÁTICA, 7., 2013, Uruguay. Actas del VII Congreso Iberoamericano de Educación Matemática. Uruguay: FISEM, 2013. p. 2856-2863. Disponible en: 〈http://www.cibem7.semur.edu.uy/7/actas/pdfs/274.pdf〉. Acceso en: 10 ago. 2012.

MATO, M.; DE LA TORRE, E. Evaluación de las actitudes hacia las matemáticas y el rendimiento académico. PNA Revista de investigación en Didáctica de la Matemática, España, v. 5, n. 1, 2010.

MCLEOD, D. Affective issues in mathematical problem solving: Some theoretical considerations. Journal for Research in Mathematics Education, USA, v. 19, n. 2, p. 134-141, 1988.

MEJÍA, Y. Mujer y educación. Él Estudia, Ella Estudia: Representaciones Sociales de las Matemáticas y de Género. En: CONGRESO IBEROAMERICANO DE EDUCACIÓN: METAS 2021, 1., 2010, Argentina. Presentación p. 1-12. Disponible en:

<http://www.chubut.edu.ar/descargas/secundaria/congreso/MUJERYEDUCACION/RLE3298_Mejia. pdf >. Acceso en: 03 jul. 2012.

MORALES, J.; MOYA, M. Tratado de psicología social. 1ª ed. v. I: Procesos básicos. España: Síntesis, 1996.

MORENO, M. El papel de la didáctica en la enseñanza del cálculo: evolución, estado actual y retos futuros. En: MAZ, A; GÓMEZ, B.; TORRALBO, M (Ed.). SIMPOSIO DE LA SOCIEDAD ESPAÑOLA DE EDUCACIÓN MATEMÁTICA, 9., 2005, Córdoba. Actas... Córdoba: SEIEM, 2005. p. 81-96.

PÉREZ, A. La formación de actitudes en la integración de alumnos con necesidades educativas especiales. Revista Mexicana de Pedagogía, México, v. 7, n. 1, p. 15-19, 1996.

RODRÍGUEZ, N. Actitudes de los estudiantes universitarios hacia la estadística. Interdisciplinaria: Revista de Psicología y Ciencias Afines, Argentina, v. 28, n. 2, p. 199-205, 2011. 
ROJAS, C.; GUACANEME, E. ¿Qué nos dicen las investigaciones en didáctica de las matemáticas sobre la enseñanza de la derivada? Revista Científica, Colombia, Edición especial, p. 201-205, 2013.

Ruiz, D. et al. Representaciones sociales en el aprendizaje de las matemáticas. Revista Educere, Venezuela, v. 15, n. 51, p. 439-449, 2011.

SÁNCHEZ-MATAMOROS, G.; GARCÍA, M.; LLINARES, S. La comprensión de la derivada como objeto de investigación en didáctica de la matemática. Revista Latinoamericana de Investigación en Matemática Educativa, México, v. 11, n. 2, p. 267-296, 2008.

SUMMERS, G. Medición de actitudes. $1^{\mathrm{a}}$ reimpresión en español. México: Trillas, 1978.

VEGA, M.; CARRILLO, J.; SOTO, J. Análisis según el Modelo Cognitivo APOS del Aprendizaje Construido del Concepto de la Derivada. BOLEMA, Brasil, v. 28, n. 48, p. 403-429, 2014.

VILANOVA, S. et al. Concepciones de los docentes sobre las Matemáticas. Su incidencia en la enseñanza y el aprendizaje. Acta latinoamericana de Matemática Educativa, México, n. 18, p. 425430, 2005.

VILLORO, L. Creer saber, conocer. $1^{\text {a }}$. ed. México: Siglo XXI, 2002.

VRANCKEN, S. et al. Dificultades relacionadas con la enseñanza y el aprendizaje del concepto de límite. Revista PREMISA, Argentina, v. 8, n. 29, p. 9-19, 2006. 